\title{
Originales
}

\section{Prevalencia de anticuerpos $\lg G$ al toxoide tetánico en trabajadores sanitarios}

\section{Prevalence of $\lg G$ antibodies to toxin tetanus in health workers}

\section{B. Pérez Bermúdez ${ }^{1}$, M. ${ }^{a}$ J. Almodóvar Carretón', Octavio Ramón de Fata ', Santiago Vila Duplá ${ }^{2}$, Carmen Martínez López ' ${ }^{\text {, A. Aranda Vizcaíno' }}$}

1. Hospital Universitario de la Princesa. Madrid. España.

2. Hospital Infantil Universitario del Niño Jesús. Madrid. España.

Recibido: 28-02-14

Aceptado: 09-08-14

\section{Correspondencia}

Brígido Pérez Bermúdez

Médico de Trabajo

Servicio de Prevención R. Laborales. Área 2.

Hospital U. de la Princesa. Madrid. España.

Correo electrónico: brigido.perez@salud.madrid.org

Resumen

Introducción: En el año 2009 el Ministerio de Sanidad y Consumo de España publica una nueva guía para la vacunación de la Difteria y Tétanos. Existen discrepancias con los tiempos de inoculación de las dosis de recuerdo con otros países y sociedades médicas.

Material y métodos: Estudio descriptivo. Las variables analizadas fueron: IgG-Toxoide Tetánico, tiempo última dosis de vacunación, dosis totales, edad, sexo, hospital, habito tabáquico e índice de masa corporal. Se construyó un modelo de Regresión Logística. Con el fin de determinar los puntos de corte más sensibles y específicos para las variables temporales (tiempo desde la última dosis y edad) se calcularon las curvas R.O.C.

Resultados: Las variables, edad $\mathrm{O}, \mathrm{R}=17,56(1,840-167,760)$, dosis de vacuna inoculadas al trabajador, $\mathrm{O}, \mathrm{R}=0,064(0,006-0,908)$ y tiempo de la última dosis de vacunación, $\mathrm{O}, \mathrm{R}=1,03(1,017-1,047)$, son las variables significativas en el análisis multivariante. Los puntos de cortes, para la edad son 54 años (Sensibilidad $\mathrm{S}=0,72$, Especificidad $\mathrm{SP}=0,77)$ y 15 años tras la última dosis años $(\mathrm{S}=0,88, \mathrm{SP}=0,86)$, por encima de este corte la probabilidad de no tener anticuerpos al Tétanos es alta.

Conclusiones: Ante un trabajador que refiere o documenta que hace más de 15 años de la ultima dosis deberíamos plantearnos la revacunación, si además este trabajador refiere no tener más de 5 dosis inoculadas y tiene más de 54 años la revacunación sería muy recomendable.

Med Segur Trab (Internet) 2014; 60 (236) 480-488

Palabras claves: Prevalencia anticuerpos tétanos, Inmunoglobulina G - Toxoide tetánico, Vacunación tétanos en adultos, Trabajadores sanitarios. 


\section{Abstract}

Introduction: In 2009 the Ministry of Health of Spain published a new guide for the vaccination of diphtheria and tetanus. There are discrepancies with the time of inoculation of the booster with other countries and medical societies. The objective of this study was to estimate the health worker prevanlencia antibody negative to tetanus toxoid and see to it associated factors.

Material and Methods: A descriptive study. The variables analyzed were: IgG - Tetanus Toxin, time last dose of vaccination, total dose, age, sex, hospital, smoking history and body mass index. Logistic regression model was constructed. In order to identify areas of more sensitive and specific cut for temporary variables (time since last dose and age) ROC curves were calculated.

Results: The variables, age O.R= 17,56 (1,840 - 167,760), inoculated vaccine doses to workers O. $R=0,064(0,006-0,908)$ and the time of the last dose of vaccination $O . R=1,03(1,017-1,047)$ are significant variables in the multivariate analysis. The cut-off points for age is 54 years (Sensitivity $S=0,72, S$ pecificity $\mathrm{SP}=0,77)$ and 15 years after the last dose $(\mathrm{S}=0,88, \mathrm{SP}=0,86)$, above this cut the likelihood of having antibodies to tetanus is high.

Conclusions: In a worker or documents referred to more than 15 years ago the last dose revaccination should ask, if this worker also reported not having more than 5 inoculated dose and has over 54 years would be highly recommended revaccination.

Med Segur Trab (Internet) 2014; 60 (236) 480-488

Keywords: Prevalence tetanus antibody, Immunoglobulin G - Tetanus toxin, Tetanus vaccination in adults, Health workers. 


\section{INTRODUCCIÓN}

En el año 2009 el Ministerio de Sanidad y Consumo de España publica una guía con la nueva pauta de vacunación para la Difteria y el Tétanos ${ }^{1}$, en consonancia con otros organismos y países como la OMS ${ }^{2,3}$, Holanda ${ }^{4}$, Australia ${ }^{5}$, etc. En esta guía se recomiendan 5 dosis hasta los 15 años y posteriormente 1 dosis en edad adulta. Sin embargo otros países continúan con la pauta de una primo-vacunación, dosis de recuerdo en la infancia y adolescencia, y posteriormente dosis de recuerdo cada 10 años a lo largo de toda la vida ${ }^{6,7}$. Algunas Sociedades medicocientíficas en España como la Sociedad de Medicina Preventiva ${ }^{8}$ en sus últimas recomendaciones, siguen abogando o recomendando recuerdos cada 10 años.

Los motivos que se consideran para recomendar esta nueva pauta están basados fundamentalmente en los estudios de seroprevalencia, características farmacocinéticas directamente relacionadas con la composición de las vacunas y por ultimo criterios costoefectivos. Así la Encuesta Nacional de seroprevalencia del año 1996 ${ }^{\circ}$, constata que el 95\% de la cohorte nacida después del año 1982 tienen títulos de anticuerpos protectores, aunque esta misma encuesta recoge que la cohorte nacida después de 1966 su seroprotección solo alcanza el $54.6 \%$. Respecto a la composición de las vacunas se argumenta que existe una diferencia sustancial de potencia inmunógena entre el toxoide «no adsorbido" y el "adsorbido», siendo este ultimo el más inmunógeno y el que se utiliza en las vacunas comercializadas en España, produciendo una duración de la inmunidad más prolongada en el tiempo. Por ultimo existe un estudio de coste efectividad del año $1993^{10}$ en el que la estrategia más costo-efectiva sería inocular solo una dosis de recuerdo alrededor de los 65 años, siempre que el adulto haya recibido más de cinco dosis previas a lo largo de la vida.

Por otro lado además de ser una vacunación sistemática en el adulto la vacunación de difteria tétanos también esta especialmente recomendada en trabajadores susceptibles de producirse heridas con frecuencia como son los trabajadores sanitarios. Es una parte fundamental en el ejercicio de nuestro trabajo adoptar las medidas de prevención necesarias para evitar desarrollo de enfermedades inmunoprevenibles en los trabajadores ${ }^{11-13}$.

Ante estas consideraciones se decidió determinar en las consultas de Medicina del Trabajo la seroprevalencia de anticuerpos IgG al toxoide tetánico con el fin de identificar trabajadores con anticuerpos negativos y analizar si existen factores asociados a esta negatividad.

\section{MATERIAL Y MÉTODOS}

Estudio descriptivo, realizado durante el año 2013. La muestra la constituyen 100 trabajadores del Hospital de Santa Cristina y del Hospital Infantil Niño Jesús de Madrid, dependientes del Servicio de Prevención de Riesgos Laborales del Hospital Universitario de La Princesa (total de trabajadores 1800). El cálculo del tamaño muestral se realizó mediante la aplicación informática EPIDAT v.3.1, asumiendo una prevalencia esperada del 5 al $10 \%$ que estimamos por experiencia propia de nuestras consultas y asumiendo un nivel de confianza del $95 \%$. El muestreo fue consecutivo entre los trabajadores que acudían a los exámenes de salud tanto periódicos como iniciales que referían o documentaban tener inoculada la última dosis hacía 10 años o más. A estos se le solicitaba en la analítica de rutina del examen de salud la IgG al toxoide tetánico. La muestra es representativa de los trabajadores del hospital que acuden a los exámenes de salud ya que se obtenían los datos en el examen de salud así como la solicitud de la serología.

La determinación de las IgG-Toxoide Tetánico, se realizó mediante el test de ELISA de la casa DRG. Instruments GMBH (Distribuidor Palex Medical S.A.). Se consideró que el test era negativo cuando se obtenían niveles inferiores a $0.10 \mathrm{IU} / \mathrm{mL}$. Se consideraron resultados dudosos valores de 0.10 a $0.15 \mathrm{I}$.U./mL. De las 100 determinaciones analizadas, 5 dieron resultados dudosos, a estos se consideraron como negativos, ya que en la práctica clínica se les recomendaría inocular una dosis de refuerzo. 
Las variables analizadas fueron: resultado de la IgG-Toxoide Tetánico, tiempo desde la última dosis de vacunación al toxoide tetánico (en meses), dosis totales recibidas (se dicotomizó en más de 5 dosis o menos), edad, sexo, centro hospitalario, habito tabáquico e índice de masa corporal (I.M.C.).

Se realizó un estudio bivariante. Para la comparación de proporciones se aplicó ji cuadrado, o test de Fisher cuando no se consideraban datos paramétricos y para variables continuas la t-student, en estas se asumió y comprobó normalidad, se consideró significativa $\mathrm{p}<0,05$. Posteriormente, se construyó un modelo multivariante mediante una Regresión Logística, bajo el enfoque predictivo mediante el método de máxima verosimilitud e introduciendo las variables en bloque. La variable dependiente fue IgG al toxoide Tetánico positivo o negativo y las variables independientes todas las citadas anteriormente. Para la variable edad se dicotomizó respecto a la mediana que fue de 50 años. La medida de asociación fue la Odds Ratio (O.R) y se calcularon sus intervalos de confianza al $95 \%$. (I.C 95\%). La bondad de ajuste se realizó mediante el test de Hosmer y Lemeshow y mediante el cálculo del área de la curva ROC (Receiver Operating Characteristic) de las probabilidades de los valores pronosticados.

Posteriormente con el fin de determinar los puntos de corte más sensibles y específicos se calcularon las curvas R.O.C. con sus áreas correspondientes y sus I.C al 95\%. Estas curvas R.O.C. se utilizaron para las variables temporales (tiempo desde la última dosis de vacunación del toxoide tetánico y edad). El análisis de los datos se realizó con el programa SPSS. v. 17.0.

\section{RESULTADOS}

La muestra fue de 100 trabajadores, obteniéndose una prevalencia de anticuerpos negativos al Toxoide Tetánico de 25 trabajadores (25\%). En la tabla I se muestra el resultado bivariante de las variables estudiadas.

Tabla I. Descriptivo y análisis bivariante. Relación de las variables analizadas respecto a existencia de anticuerpos al Toxoide Tetánico

\begin{tabular}{|c|c|c|c|}
\hline Variables & $\begin{array}{c}\text { IgG-Negativas } \\
25(25 \%)\end{array}$ & $\begin{array}{l}\text { IgG-Positivas } \\
75(75 \%)\end{array}$ & $\mathbf{P}$ \\
\hline EDAD $^{(*)}$ & $57,2(8,1)$ años & $46,1(11,2)$ años & 0,000 \\
\hline SEXO & & & $\mathbf{0 , 0 2 3} 3^{(* *)}$ \\
\hline Hombre & $8(50,0 \%)$ & $8(50,0 \%)$ & \\
\hline Mujer & $17(20,2 \%)$ & $67(79,8 \%)$ & \\
\hline CENTRO HOSPITALARIO & & & 0,554 \\
\hline H. I. Niño Jesús & $11(28,2 \%)$ & $28(71,8 \%)$ & \\
\hline H. Sta. Cristina & $14(23,0 \%)$ & $47(77,0 \%)$ & \\
\hline \multicolumn{4}{|l|}{ SANITARIO } \\
\hline Sí Sanitario & $22(24,7 \%)$ & $67(75,3 \%)$ & $1,00^{(* *)}$ \\
\hline No Sanitario & $3(27,3 \%)$ & $8(72,7 \%)$ & \\
\hline TABACO & & & $0,388^{(*)}$ \\
\hline Fumador & $3(10,8 \%)$ & $16(84,2 \%)$ & \\
\hline No fumador & $22(27,2 \%)$ & $59(72,8 \%)$ & \\
\hline I.M.C. ${ }^{(*)}$ & $25,2(3,3)$ & $24,4(3,6)$ & 0,303 \\
\hline DOSIS INOCULADAS & & & $0,007^{(* *)}$ \\
\hline Más de 5 dosis & $19(20,9 \%)$ & $72(79,1 \%)$ & \\
\hline Menos de 5 dosis & $6(66,7 \%)$ & $3(33,3 \%)$ & \\
\hline MESES DESDE ULT. DOSIS ${ }^{(*)}$ & $283,0(103,5)$ & $154,2(48,1)$ & $\mathbf{0 , 0 0 0}$ \\
\hline
\end{tabular}

(*) Media (desviación típica).

(**) Estadístico exacto de Fisher. 
Como se puede observar en la Tabla I, las variables edad, sexo, dosis inoculadas anteriormente a la determinación de los anticuerpos y el tiempo transcurrido desde la última dosis inoculada, son significativas en el análisis bivariante.

Para comprobar que en las variables analizadas no existen variables confusoras se construyó un modelo multivariante por medio de una Regresión Logística cuyos resultados se muestran en la tabla II.

Tabla II. Regresión Logística. Variable dependiente existencia o no, de anticuerpos al Toxoide Tetánico

\begin{tabular}{lccc}
\hline \multicolumn{1}{c}{ Variables } & $\mathbf{P}$ & $\mathbf{O . R}$ & I.C. 95\% \\
\hline Tiempo desde la ultima dosis & $\mathbf{0 , 0 0 0}$ & $\mathbf{1 , 0 3 2}$ & $\mathbf{( 1 , 0 1 7}-\mathbf{1 , 0 4 7 )}$ \\
Dosis de Vacunas & $\mathbf{0 , 0 4 1}$ & $\mathbf{0 , 0 6 4}$ & $\mathbf{( 0 , 0 0 6}-\mathbf{0 , 9 0 8 )}$ \\
Edad & $\mathbf{0 , 0 1 3}$ & $\mathbf{1 7 , 5 6 8}$ & $\mathbf{( 1 , 8 4 0 ~ - ~ 1 6 7 , 7 6 0 )}$ \\
Sexo & 0,165 & 0,156 & $(0,011-2,152)$ \\
Sanitario & 0,705 & 0,613 & $(0,048-7,759)$ \\
Centro Hospitalario & 0,918 & 0,904 & $(0,133-6,149)$ \\
Fumar & 0,187 & 0,130 & $(0,006-2,689)$ \\
I.M.C & 0,224 & 0,819 & $(0,564-1,130)$ \\
\hline
\end{tabular}

Las variables edad, dosis de vacunas inoculadas al trabajador y el tiempo en meses desde la ultima dosis de vacuna antitetánica son las variables significativas, de tal forma que el hecho de ser mayor de 50 años, implica una O.R. 17,1 veces más probable que la determinación de los anticuerpos al tétanos sean negativos. Por otra parte, el hecho de tener inoculadas mas de cinco dosis hace menos probable tener anticuerpos negativos, aproximadamente dos veces menos probable, con una O.R. de 1,56 (1 / 0,64). Por ultimo, por cada mes que pase desde la última dosis aumentaría la O.R un 3,0\% la probabilidad de no tener anticuerpos.

Al observar que estas variables han sido las significativas en la muestra analizada, se decidió crear puntos de cortes en la variables «edad" y en el «tiempo desde la última dosis» de inoculación aplicando curvas R.O.C. Se considera al trabajador con IgG negativa como estado "no deseado" (enfermo) y al que tiene anticuerpos, como trabajador en estado "deseado" (sano) y sobre las variables continuas (edad y tiempo desde la última dosis) establecemos los puntos de corte donde la Sensibilidad (probabilidad de clasificar correctamente a un trabajador con IgG negativas) y Especificidad (probabilidad de clasificar correctamente a un trabajador con IgG positivas) son más altas. Los resultados se muestran en la tabla III.

Tabla III. Punto de Corte de las variables Edad y Tiempo desde la última dosis con la Sensibilidad, Especificidad calculada

\begin{tabular}{lccc}
\hline \multicolumn{1}{c}{ Variable } & PUNTO DE CORTE & SEN & ESP \\
\hline Edad & 54 años & 0,72 & 0,77 \\
Tiempo última dosis & 189 meses (15,7 años) & 0,88 & 0,86 \\
\hline
\end{tabular}

Las curvas ROC así como su área de la edad se muestra en el gráfico 1. Y la del tiempo desde la última dosis en el gráfico 2 . 


\section{Gráfico 1. Curva ROC edad}

\section{Curva COR}

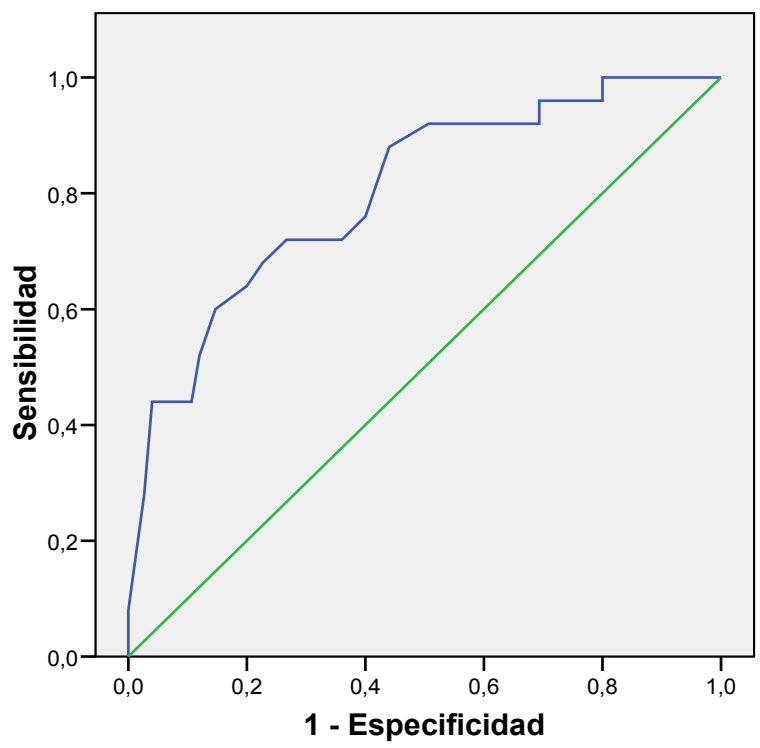

Área $=0,803(0,703-0,903)$

Gráfico 2. Curva ROC tiempo desde última dosis

Curva COR

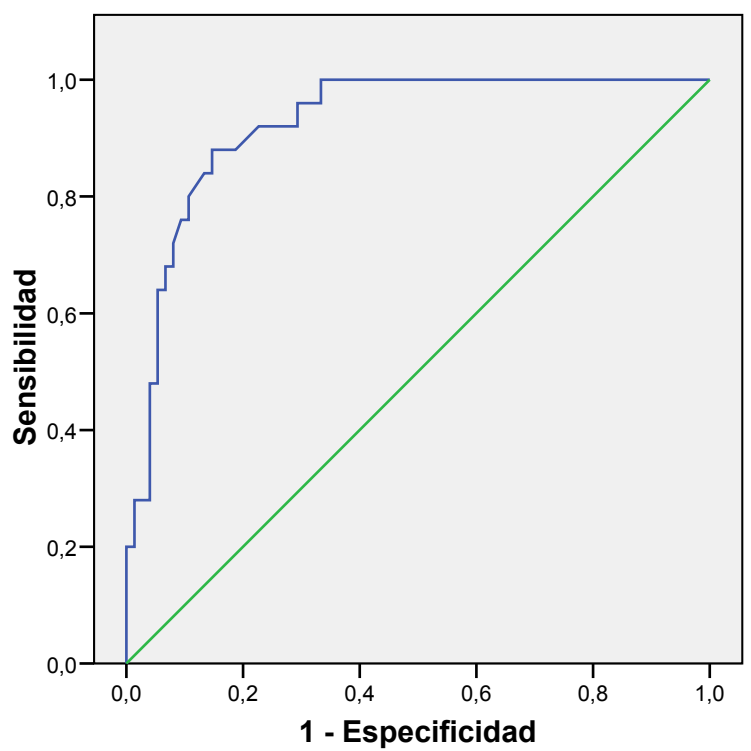

Área $\mathrm{ROC}=0,924(0,872-0,976)$

Recordemos que la interpretación de este último gráfico y su área es que si obtenemos un par de trabajadores, uno que se hubiese inoculado la ultima dosis en un tiempo inferior a 189 meses (15 años y medio) y otro que se hubiese inoculado la ultima dosis más de 189 meses, la probabilidad de clasificar correctamente como trabajador con IgG negativa o positiva alcanzaría el $92,4 \%$. 


\section{DISCUSIÓN Y CONCLUSIONES}

Este tipo de estudios se abordan desde la óptica de los análisis de supervivencia pero en la práctica de nuestras consultas la toma de decisión respecto a la idoneidad de inocular dosis de recuerdo de vacuna antitetánica, sin el apoyo del laboratorio, se basa fundamentalmente en variables temporales. A un trabajador le resulta más fácil recordar la última inoculación de la vacuna, que las dosis inoculadas a lo largo de su vida. Por tanto la justificación de este estudio y el análisis empleado, se enfoca desde la necesidad de obtener para la práctica diaria de las consultas de Vigilancia de la Salud, puntos de corte en las variables «edad" y "tiempo desde última inoculación» tras comprobar que son las variables que se asocian significativamente con la ausencia de anticuerpos al tétanos en nuestra población trabajadora.

En el estudio existe un sesgo de información, aproximadamente el $50 \%$ de los trabajadores estudiados en la muestra no presentaron el carnet de vacunación o se carecía de registros de inoculación de la vacuna antitetánica en su historia laboral, por tanto los datos se han obtenido basándose en la memoria de los trabajadores con un interrogatorio dirigido dentro del examen de salud, enfatizando en recordar el tiempo de la última inoculación de vacuna antitetánica, bien por heridas o por incorporación en algún puesto de trabajo que estuviese protocolizado está vacunación, así como el número de dosis inoculadas en su vida. Es evidente que las variables «tiempo desde la ultima dosis»y "dosis inoculadas" podrían ser datos sesgados aunque también podrían considerarse como un sesgo "no diferencial" ya que algunos registros estarían sobreestimados al igual que otros serían infraestimados.

Se recogieron las variables de obesidad y hábito tabáquico cuando se inocularon la última dosis, porque algunos artículos ${ }^{14}$ los relacionan con una menor respuesta a la vacunación, en estas variables no se observan diferencias significativas en este estudio.

Aunque en el análisis bivariante ser hombre parece tener una mayor proporción de IgG-negativas, esta significación no se confirma en el análisis multivariante, no obstante a pesar de la escasa proporción de hombres en el ámbito sanitario llama la atención que proporcionalmente existan más trabajadores con anticuerpos negativos teniendo en cuenta que en muchos estudios de seroprevalencia ocurre lo contrario, existiendo una mayor prevalencia de anticuerpos negativos en las mujeres que algunos autores relacionan con el hecho de que los varones al tener que realizar el Servicio Militar Obligatorio, se les inoculaba dosis de refuerzo e incluso en muchas ocasiones hasta una pauta completa de vacunación al Tétanos y a la Difteria.

Otro punto importante a destacar en esta discusión es que se observa una prevalencia muy elevada (25\%) de trabajadores que presentan títulos negativos de anticuerpos IgG al tétanos. Esto puede hacer sospechar que quizás esta correlación que se admite entre este tipo de test ELISA con el test del estudio de linfocitos (memoria celular) no sea tan evidente ya que a pesar de la elevada prevalencia de adultos sin IgG-tetánicas, no se corresponde con la incidencia de enfermedad tetánica observada en la población. Posiblemente debe existir una inmunidad celular no cuantificable que perdura más de lo que registra estos tipos de test.

Como se ha referido anteriormente, el trabajo desarrollado se elaboró con el fin de estudiar en el ámbito sanitario la prevalencia de trabajadores con anticuerpos negativos al tétanos y secundariamente desarrollar un procedimiento de actuación ante las distintas guías editadas y hasta cierto punto contradictorias, fundamentalmente en lo referente al tiempo de inoculación de la dosis de recuerdo, no solo de distintos organismos y sociedades científicas españoles sino también entre países. Como sabemos existen ciertos consensos que aconsejan dosis de refuerzo cada 10 años sin tener en cuenta las dosis totales inoculadas en la infancia-adolescencia, y otros como la guía del Ministerio de Sanidad de España que aconseja no inocular dosis de refuerzo hasta los 60 años si ha recibido anteriormente más de cinco dosis. En este estudio se observa que existe una diferencia significativa entre los trabajadores que tienen más 
de cinco dosis y los que no las tienen inoculadas, pero también se observa significativamente que al aumentar el tiempo desde la última inoculación la probabilidad de no tener anticuerpos es mayor independientemente de las dosis inoculadas. Siendo a partir de los aproximadamente 15 años desde la ultima dosis cuando la probabilidad de hallar anticuerpos negativos o dudosos es casi, 6 veces más (Razón de verosimilitud positiva= Sensibilidad / 1-Especificidad) que si es menos de 15 años. Por tanto en un adulto que su última inoculación se produjo más de 15 años se debería valorar la posibilidad de vacunación.

En el análisis realizado la edad es una variable significativa, observando más probabilidad de encontrar trabajadores con anticuerpos negativos entre los que son mayores de 54 años. No obstante se debería de valorar la posibilidad de no esperar a los 60 años si el trabajador tras interrogatorio de la ultima fecha de la inoculación de la vacuna al tétanos refiere más de 15 años y tiene más de 54 años, para inocular una dosis de recuerdo.

Quisiéramos realizar una reflexión respecto a la vacuna del tétanos en los trabajadores sanitarios, que es donde realizamos nuestra labor. En los últimos años se está poniendo mucha atención en la importancia e idoneidad de la correcta vacunación a la bordetella pertussis por el aumento de incidencia de la Tos ferina en España, si en el año 2010 existía una incidencia de aproximadamente 2 casos por 100,000 habitantes, en el año 2011 se ha pasado a una Incidencia de 7 casos por 100,000 habitantes. Los últimos consensos ${ }^{15,16}$ aconsejan inocular dosis de refuerzo a los trabajadores sanitarios y más concretamente a los sanitarios relacionados con el cuidado y la atención de los niños, como prolongación de la estrategia "del nido", consistente en vacunar a padres y cuidadores (en guarderías y centros sanitarios) de niños menores de tres años, considerando algunos autores que dicha vacunación debería extenderse a todos los trabajadores sanitarios. Según estudios la duración de los anticuerpos frente al toxoide de la bordetella pertussis es como máximo de 10 años ya que la vacunación no ofrece inmunidad duradera y por tanto hace recomendable dosis de refuerzo tras estos periodos de tiempo ${ }^{17}$. En España la inoculación del toxoide a la bordetella pertussis está unida a la vacuna Td de forma indisoluble, existe la vacuna combinada de Difteria, Tétanos y Pertusis (Tdpa) siendo esta la única composición comercializada en España, no existiendo un preparado comercial con la vacuna a la bordetella pertussis exclusivamente. Por tanto extremar y valorar con atención la conveniencia de inocular las dosis de refuerzo de Tétanos parece una valoración secundaria para trabajadores sanitarios teniendo en cuenta que la periodicidad de la dosis de refuerzo en los trabajadores sanitarios lo marcará la permanecía de la inmunidad frente a la bordetella pertussis.

Como conclusión, podemos decir que en base a estos resultados, ante un trabajador que refiere o documenta que hace más de 15 años de la ultima dosis se debería plantear la revacunación al toxoide tetánico, si además este trabajador refiere no tener más de 5 dosis inoculadas y ser mayor de 54 años la revacunación sería muy recomendable.

\section{REFERENCIAS BIBLIOGRÁFICAS}

1. Ministerio de Sanidad y Consumo de España. Vacunación en adultos. Recomendaciones vacunación de difteria y tetános. Actualización 2009. Disponible en: https://www.msssi.gob.es/ciudadanos/ proteccionSalud/vacunaciones/docs/TetanosDifteria_2009.pdf

2. Tetanus vaccine. WHO Position Paper. Weekly Epidemiological Record 2006; 81:198-208. Disponible en http://www.who.int/wer/2006/wer8120.pdf

3. Borrow R, Balmer P, Roper M. Immunological basis for immunization series. Module 3: Tetanus 2006 Update. World Health Organization 2006. Immnuni-zation, Vaccines and Biologicals. Disponible en:

http://www.who.int/vaccines-documents/DocsPDF07/869.pdf

4. De Melker HE, van den Hof S, Berbers GA, Nagelk-erke NJ, Rümke HC, Conyn-van Spaendonck MA. A population-based study on tetanus antitoxin levels in The Netherlands. Vaccine 18 (2000): 100-108. 
5. Australian Department of Health and Ageing. The Australian Immunisation Handbook 9th Edition. Part 3.21. Tetanus. Disponible en:

http://www.health.gov.au/internet/immunise/publishing.nsf/Content/Handbook-tetanus.

6. Institut national de prévention et d'éducation à la santé (INPES). Guide des vaccinations. Edition 2008. Disponible en:

http://www.inpes.sante.fr/CFESBases/catalogue/pdf/1133.

7. Centers for Disease Control and Prevention. Recommended Adult Immunization Schedule -United States, October 2007-September 2008. MMWR 2007 October 19; 56(41): Q1-4.

8. Domínguez V, Castro, I. Vacunación antitetánica.. Consenso 2009. Comité de Vacunas de la Sociedad Española de Medicina Preventiva, Salud Pública e Higiene. Capítulo 2. Vol XV, N. ${ }^{\circ}$ 3, 2009.

9. Pachón I, Amela C, de Ory F. Age specific sero-prevalence of poliomyelitis, diphtheria and tetanus antibodies in Spain. Epidemiol Infect 2002; 129(3): 535-41.

10. Balestra DJ, Littenberg B. Should adult tetanus immunization be given as a single vaccination at age 65 ? A cost-effectiveness analysis. J Gen Intern Med 1993 Aug; 8 (8): 405-12.

11. Ley 31/1995, de 8 de noviembre de Prevención de Riesgos Laborales. B.O.E. n. ${ }^{\circ}$ 69. 10/11/95.

12. Real Decreto 664/1997, de 12 de mayo, sobre la protección de los trabajadores contra los riesgos relacionados con al exposición agentes biológicos durante el trabajo. B.O.E., n. ${ }^{\circ}$ 124. 24/05/97.

13. Sáenz-González MC, Hernández-García I. Vacunaciones en el medio laboral. Actualización. Rev Esp Quimioter 2013;26 (4):287-297.

14. Tolosa Martínez N., Tenías Burillo J.M, Pérez Bermúdez B, Sanchís Álvarez J.B. Factores asociados a una respuesta inadecuada a la vacunación contra la hepatitis B en personal sanitario Rev Esp Salud Pública 1998. 77: 509-5 15

15. Campins M, Moreno-Perez D, Gil-de Miguel A, et all. Tos ferina en España. Situación epidemiológica y estrategias de prevención y control. Recomendaciones del Grupo de Trabajo de Tos ferina. Enferm Infecc Microbiol Clin. 2013;31(4):240-253

16. Grupo de Trabajo Tos Ferina 2012 de la Ponencia de Programas y Registro de Vacunaciones. Revisión del programa de vacunación frente a tos ferina en España. Comisión de Salud Pública del Consejo Interterritorial del Sistema Nacional de Salud. Ministerio de Sanidad, Servicios Sociales e Igualdad, 2013: 14-20 (M. Campins et al/Enferm Infecc Microbiol Clin.2013; 31(4):240-253

17. Ward JI, Cherry JD, Chang SJ, Partridge S, Lee H,Treanor J, et al. Efficacy of an acellular pertussis vaccine among adolescents and adults. NEnglJMed. 2005;353:1555-1563.

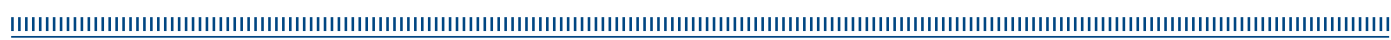

